\title{
INTRINSIC METRICS ON COMPLEX MANIFOLDS
}

\author{
BY SHOSHICHI KOBAYASHI ${ }^{1}$
}

Communicated by S. Smale, January 17, 1967

1. Definition of intrinsic pseudometric. Let $M$ be a (connected) complex manifold. We shall define a pseudometric $d$ on $M$ in a natural manner so that it depends only on the complex structure of $M$ and nothing else.

Let $D$ be the open unit disk in the complex plane and $\rho$ the distance on $D$ defined by the Poincaré-Bergman metric of $D$. Given two points $p$ and $q$ of $M$, choose the following objects:

(1) points $p=p_{0}, p_{1}, \cdots, p_{k-1}, p_{k}=q$ of $M$ and

(2) points $a_{1}, \cdots, a_{k}, b_{1}, \cdots, b_{k}$ of $D$ and holomorphic mappings $f_{1}, \cdots, f_{k}$ of $D$ into $M$ such that $f_{i}\left(a_{i}\right)=p_{i-1}$ and $f_{i}\left(b_{i}\right)=p_{i}$ for $i=1, \cdots, k$.

For each choice of points and mappings satisfying (1) and (2), consider the number $\rho\left(a_{1}, b_{1}\right)+\cdots+\rho\left(a_{k}, b_{k}\right)$. Let $d(p, q)$ be the infimum of the numbers obtained in this manner for all possible choices. It is easy to verify that $d$ is a pseudometric on $M$ in the sense that

$$
d(p, q) \geqq 0, \quad d(p, q)=d(q, p), \quad d(p, q)+d(q, r) \geqq d(p, r)
$$

for $p, q, r \in M$. The following two propositions are immediate from the definition of $d$.

Proposition 1. Let $M$ and $N$ be two complex manifolds and $d_{M}$ and $d_{N}$ the intrinsic pseudometrics of $M$ and $N$. Then every holomorphic mapping $f: M \rightarrow N$ is distance-decreasing in the sense that

$$
d_{M}(p, q) \geqq d_{N}(f(p), f(q)) \text { for } p, q \in M .
$$

In particular, every holomorphic transformation of $M$ is distancepreserving with respect to $d_{M}$.

Proposition 2. For the complex Euclidean space $C^{n}$, the pseudometric $d$ is trivial, i.e., $d(p, q)=0$ for all $p, q \in C^{n}$.

The following proposition follows from the Schwarz-Pick lemma.

Proposition 3. For the unit disk $D$, the pseudometric $d$ coincides with the distance $\rho$ defined by the Poincaré-Bergman metric.

${ }^{1}$ Supported partially by NSF Grant GP-5798. 
The following theorem follows from a generalized Schwarz-Pick lemma (see [3] and [4]). ${ }^{2}$

Theorem 4. Let $M$ be a hermitian manifold whose holomorphic sectional curvature is negative and bounded away from zero. Then its pseudometric $d$ is a metric, i.e., $d(p, q)=0$ implies $p=q$.

Theorem 4 applies to all bounded domains of $C^{n}$ as well as to all Riemann surfaces of hyperbolic type.

2. Relationship with Carathéodory metric. Following Carathéodory [2] we define another pseudometric $d^{\prime}$ on a complex manifold $M$. Given two points $p$ and $q$ of $M$, let $d^{\prime}(p, q)$ be the supremum of $\rho(f(p), f(q))$ for all holomorphic mappings $f$ of $M$ in to the unit disk $D$.

It is easy to see that Propositions 1, 2 and 3 above hold also for the Carathéodory pseudometric $d^{\prime}$. A necessary and sufficient condition for $d^{\prime}$ to be a metric on $M$ is that there are sufficiently many bounded holomorphic functions on $M$ so that they separate the points of $M$.

The following proposition is immediate from Propositions 1 and 3 and shows that whenever the Carathéodory pseudometric $d^{\prime}$ is a metric, our pseudometric $d$ is also a metric.

Proposition 5. For any complex manifold $M, d$ is greater than or equal to $d^{\prime}$, i.e.,

$$
d(p, q) \geqq d^{\prime}(p, q) \quad \text { for } p, q \in M .
$$

3. Applications. The following theorem which follows from Propositions 1 and 2 and Theorem 4 may be considered as a generalization of Picard Theorem which states that an entire function with more than one finite lacunary value reduces to a constant function.

Theorem 6. Let $M$ be an n-dimensional complex manifold which admits a hermitian metric whose holomorphic sectional curvature is negative and bounded away from zero. Then every holomorphic mapping $f$ of $C^{m}$ into $M$ is a constant map.

Corollary. The complex Euclidean space $C^{m}$ does not admit a hermitian metric whose holomorphic sectional curvature is negative and bounded away from zero.

The condition "bounded away from zero" is essential. In fact, $C$

${ }^{2}$ The results proved for Kähler manifolds in [4] hold for hermitian manifolds (with respect to the hermitian connection in the sense of Chern). Proofs there remain valid in the hermitian case. 
(and hence $C^{m}$ ) admits a complete Kähler metric of negative holomorphic sectional curvature, e.g.,

$$
(1+z \bar{z}) d z d \bar{z} .
$$

THEOREM 7. Let $M$ be a hermitian manifold whose holomorphic sectional curvature is negative and bounded away from zero. Then the group of holomorphic transformations of $M$ is a Lie group with compact isotropy subgroups (with respect to the compact-open topology).

In fact, the group in question is a closed subgroup of the group of isometries of $M$ with respect to the intrinsic metric $d$ introduced above. By a classical theorem of Van Dantzig and van der Waerden (see Theorem 4.7 and Corollary 4.8 of Chapter I in [5]) the group of isometries of a locally compact metric space is locally compact and its isotropy subgroups are all compact with respect to the compactopen topology. Theorem 7 follows now from a well-known theorem of Bochner-Montgomery [1].

Corollary 8. If, in Theorem 7, $M$ is moreover compact, then the group of holomorphic transformations of $M$ is a finite group.

The results in this section have been obtained by $\mathrm{Wu}[6]$ using the notion of normal families.

\section{BIBLIOGRAPHY}

1. S. Bochner and D. Montgomery, Locally compact groups of differentiable transformations, Ann. of Math. 47 (1946), 639-653.

2. C. Carathéodory, Über das Schwarzsche Lemma bei analytischen Funktionen von zwei komplexen Veränderlichen, Math. Ann. 97 (1926), 76-98.

3. H. Grauert and H. Reckziegel, Hermitesche Metriken und normale Familien holomorphic Abbildungen, Math. Z. 89 (1965), 108-125.

4. S. Kobayashi, Distance, holomorphic mappings and the Schwarz's lemma, J. Math. Soc. Japan (to appear).

5. S. Kobayashi and K. Nomizu, Foundations of differential geometry, Interscience Tracts No. 15, Interscience, New York, 1963.

6. H. H. Wu, Normal families of holomorphic mappings and the theorem of Bloch in several complex variables (to appear).

University of California, Berkeley 